\title{
Nuclear multifragmentation and fission: similarity and differences
}

\author{
V. Karnaukhov ${ }^{1,1}$, H. Oeschler ${ }^{2}$, S. Avdeyev ${ }^{1}$, V. Rodionov ${ }^{1}$, \\ V. Kirakosyan ${ }^{1}$, A. Simonenko ${ }^{1}$, P. Rukoyatkin ${ }^{1}$, A. Budzanowski ${ }^{3}$, \\ W. Karcz ${ }^{3}$, I. Skwirczynska ${ }^{3}$, B. Czech $^{3}$, L. Chulkov ${ }^{4}$, E. Kuzmin ${ }^{4}$, \\ E. Norbeck ${ }^{5}$, A. Botvina ${ }^{6}$ \\ ${ }^{1}$ Joint Institute for Nuclear Research, 141980 Dubna, Russia \\ ${ }^{2}$ Institut für Kernphysik, Darmstadt University of Technology, 64289 Darmstadt, Germany \\ ${ }^{3}$ H. Niewodniczanski Institute of Nuclear Physics, 31-342 Cracow,Poland \\ ${ }^{4}$ Kurchatov Institute, 123182 Moscow, Russia \\ ${ }^{5}$ University of Iowa, Iowa City, IA 52242, USA \\ ${ }^{6}$ Institute for Nuclear Research, 117312 Moscow, Russia
}

Thermal multifragmentation of hot nuclei is interpreted as the nuclear liquid-fog phase transition deep inside the spinodal region. The experimental data for $\mathrm{p}(8.1 \mathrm{GeV})+\mathrm{Au}$ collisions are analyzed. It is concluded that the decay process of hot nuclei is characterized by two size parameters: transition state and freeze-out volumes. The similarity between dynamics of fragmentation and ordinary fission is discussed. The IMF emission time is related to the mean rupture time at the multi-scission point, which corresponds to the kinetic freeze-out configuration.

\section{Introduction}

The study of the highly excited nuclei is one of the challenging topics of nuclear physics, giving access to the nuclear equation of state for temperatures below $T_{c}$ - the critical temperature for the liquidgas phase transition. The main decay mode of hot nuclei is a copious emission of intermediate mass fragments (IMF), which are heavier than $\alpha$-particles but lighter than fission fragments. An effective way to produce hot nuclei is via heavy-ion collisions. But in this case the heating of nuclei is accompanied by compression, strong rotation, and shape distortion, which influence the decay properties of excited nuclei. One gains simplicity, and the picture becomes clearer, when light relativistic projectiles (protons, antiprotons, pions) are used. In this case, fragments are emitted by only one source - the slowly moving target spectator. Its excitation energy is almost entirely thermal. Light relativistic projectiles provide a unique possibility for investigating thermal multifragmentation. The decay properties of hot nuclei are well described by statistical models of multifragmentation (SMM and MMMC [1, 2]). This is an indication, that the system is thermally equilibrated or close to that. For the case of peripheral heavy-ion collisions the partition of the excited system is also governed by heating.

The van der Walls equation can be used with nuclear matter because of the similarity of the nucleonnucleon force to the force between molecules in a classical gas [3, 4, 5]. In both cases there exists a region in the PVT diagram corresponding to a mixture of liquid and gas phases. This region can contain unstable, homogeneous matter for short times. In a classical gas this can be achieved by cooling through the critical point. In the nuclear case this can be achieved by a sudden expansion of the liquid phase at a temperature well below the critical temperature. The separation of the homogeneous matter into a mixture of stable liquid and gas is called spinodal decomposition. One can imagine that a hot nucleus

\footnotetext{
${ }^{1}$ Email address: karna@jinr.ru
} 
(at $T=7-10 \mathrm{MeV}$ ) expands due to thermal pressure and enters the unstable region. Due to density fluctuations, a homogeneous system is converted into a mixed phase consisting of droplets (IMF) and nuclear gas interspersed between the fragments. Thus the final state of this transition is a nuclear fog [5]. Note that classical fog is unstable, it condensates finally into bulk liquid. The charged nuclear fog is stable in this respect. But it explodes due to Coulomb repulsion and is detected as multifragmentation. It is more appropriate to associate the spinodal decomposition with the liquid-fog phase transition in a nuclear system rather than with the liquid-gas transition [6, 8]. This scenario is supported by a number of observations; some of them are the following:

(a) the density of the system at break-up is much lower than the normal one $\rho_{0}[$ [ ;

(b) the mean life-time of the fragmenting system is very small $(\approx 50 \mathrm{fm} / \mathrm{c})[9$;

(c) the break-up temperature is significantly lower than $T_{c}$, the critical temperature for the liquid-gas phase transition [6, 7].

In this paper we concentrate on the dynamics of thermal multifragmentation and its similarity to ordinary fission. This similarity was noted first by Lopez and Randrup in their statistical theory of multifragmentation [10, 11]. First of all, there are two characteristic volumes (or configurations) for both processes. Secondly, the time scale characterizations for fragmentation and fission are similar with respect to their ingredients.

Experimental data have been obtained using the $4 \pi$-device FASA installed at the external beam of the Nuclotron (Dubna). At present, the setup consists of twenty five $d E-E$ telescopes surrounded by a fragment multiplicity detector, which is composed of 58 thin $\mathrm{CsI}(\mathrm{Tl})$ scintillation counters.

\section{Two characteristic volumes in thermal multifragmentation}

Traditionally, in statistical models 1, 2, multifragmentation is characterized by just one size parameter - the freeze-out volume, $V_{f}$. There are a number of papers with experimental estimations of this characteristic volume, but the values obtained deviate significantly. A mean freeze-out volume $\sim 7 V_{0}\left(V_{0}=\right.$ volume at normal density) was found in ref. [12] from the average relative velocities of the IMFs for ${ }^{4} \mathrm{He}(14.6 \mathrm{MeV})+\mathrm{Au}$ collisions. In paper [13] the nuclear caloric curves were considered within the Fermi-gas model to extract average nuclear densities for different systems. It was found that $V_{f} \approx 2.5 V_{0}$ for the medium and heavy masses. In ref. 14 the mean IMF kinetic energies were analyzed for $\mathrm{Au}+\mathrm{Au}$ collisions at $35 \cdot \mathrm{A} \mathrm{MeV}$. The freeze-out volume was found to be $\sim 3 V_{0}$. The average source density for the fragmentation in the $8.0 \mathrm{GeV} / c \pi^{-}+\mathrm{Au}$ interaction was estimated to $\mathrm{be} \approx(0.25-0.30) \rho_{0}$, at $\mathrm{E}^{*} / \mathrm{A} \sim 5 \mathrm{MeV}$ from the moving-source-fit Coulomb parameters [15, 16.

In our paper [8], the data on the charge distribution and kinetic energy spectra of IMFs produced in $p(8.1 \mathrm{GeV})+\mathrm{Au}$ collisions were analyzed using the combined INC +Exp+SMM model. The events with IMF multiplicity $M \geq 2$ were selected. The results obtained are shown at fig.1. It was shown that one should use two volume (or density) parameters to describe the process of multifragmentation, not just one as in the traditional approach. The first, $V_{t}=(2.6 \pm 0.3) V_{0}$ (or $\left.\rho_{t} \approx 0.38 \rho_{0}\right)$, corresponds to the stage of pre-fragment formation. Strong interaction between pre-fragments is still significant at this stage. The second one, $V_{f}=(5 \pm 1) V_{0}$, is the kinetic freeze-out volume. It is determined by comparing the measured fragment energy spectra with the model predictions using multi-body Coulomb trajectories. The calculations have been started with placing all charged particles of a given decay channel inside the freeze-out volume $V_{f}$. In this configuration the fragments are already well separated from each other, they are interacting via the Coulomb force only. Actually, the system at freeze-out belongs to the mixed phase sector of the phase diagram, with a mean density that is five times less than normal nuclear 


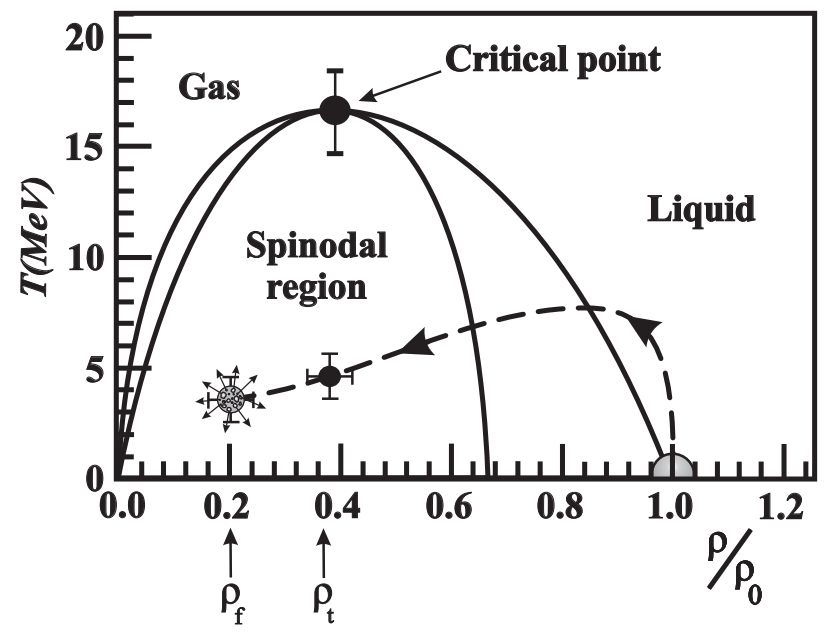

Figure 1: Proposed spinodal region for the nuclear system. The experimental points were obtained by the FASA collaboration. The arrow line shows the way from the starting point at $T=0$ and normal nuclear density $\rho_{0}$ to the break-up point at $\rho_{t}$ and to the kinetic freeze-out at the mean density $\rho_{f}$. Critical temperature is estimated in [7].

density. The first characteristic volume, $V_{t}$, was obtained by analyzing the IMF charge distributions, $Y(\mathrm{Z})$, within the SMM model with a free size parameter $k$. For simplicity, the dependence of the charge distribution on the critical temperature $T_{c}$ was neglected in this analysis.

Recently, we performed a more sophisticated consideration of $Y(\mathrm{Z})$ with two free parameters, $V_{t}$ and $T_{c}$. A comparison of the data with the calculations was done for the range $3<\mathrm{Z}<9$, in which minimal systematic errors were expected. Figure 2 shows $\chi^{2}$ for comparison of the measured and calculated IMF charge distributions as a function of $V_{t} / V_{0}$ for different values of the critical temperature. The minimum value of $\chi^{2}$ decreases with increasing $T_{c}$ in the range from 15 to $19 \mathrm{MeV}$ and saturates after that. The corresponding value of $V_{t} / V_{0}$ increases from 2.4 to 2.9. The measured IMF charge distribution is well reproduced by SMM with $V_{t}$ in the range (2.5-3.0) $V_{0}$, which is close to the value obtained in [8]. As for the kinetic freeze-out volume, the present value coincides with the one given in [8], but its uncertainty is only half as much because the estimated systematic error is less: $V_{f}=(5.0 \pm 0.5) V_{0}$.

Our previous conclusion about the value of the critical temperature [6] 7] is also confirmed: $T_{c}$ exceeds $15 \mathrm{MeV}$. Note, this value is twice as large as estimated in $[17,18]$ with the Fisher droplet model. This contradiction is waiting for further efforts to clarify the point.

\section{Comparison of multifragmentation and fission dynamics}

The occurrence of two characteristic volumes for multifragmentation has a transparent meaning. The first volume, $V_{t}$, corresponds to the fragment formation stage at the top of the fragmentation barrier. Here, the properly extended hot target spectator transforms into closely packed pre-fragments. The final channel of disintegration is completed during the evolution of the system up to the moment, when receding and interacting pre-fragments become completely separated at $V_{f}$. This is just as in ordinary fission. The saddle point (which has a rather compact shape) resembles the final channel of fission having already a fairly well defined mass asymmetry. Nuclear interaction between fission pre-fragments 


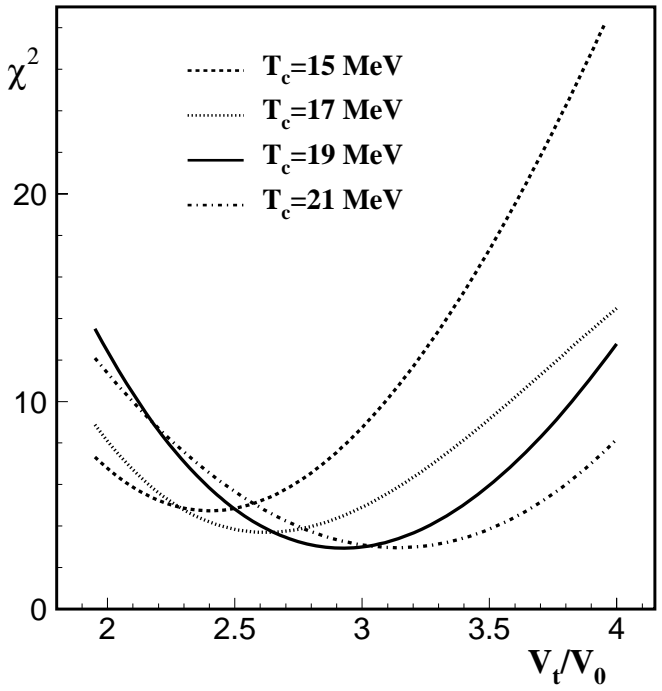

Figure 2: Value of $\chi^{2}$ as a function of $V_{t} / V_{0}$ for comparison of the measured and model predicted IMF charge distributions. The calculations with the $\mathrm{INC}^{*}+\mathrm{SMM}$ combined model was performed under the assumption of two free parameters: $V_{t}$ - the effective volume at the stage of pre-fragment formation, and $T_{c}$ - the critical temperature for the liquid-gas phase transition.

ceases after the descent of the system from the top of the barrier to the scission point. In the papers by Lopez and Randrup [10, 11] the similarity of the two processes was used to develop a theory of multifragmentation based on a generalization of the transition-state approximation first suggested by Bohr and Wheeler in 1939. The transition states are located at the top of the barrier or close to it. The phase space properties of the transition states are decisive for the further fate of the system, for specifying the final channel. No size parameters are used in the theory.

Being conceptually similar to the approach of ref. [10, 11, the statistical multifragmentation model (SMM) uses a size parameter that can be determined by fitting to data. The size parameter obtained from the IMF charge distribution can hardly be called a freeze-out volume. This is the transition state volume, corresponding to the top of the fragmentation barrier (see fig.3). The freeze-out volume, $V_{f}$, corresponds to the multi-scission point, when fragments became completely separated and start to be accelerated in the common electric field. In the statistical model (SMM) used, the yield of a given final channel is proportional to the corresponding statistical weight. This means that the nuclear interaction between pre-fragments is neglected when the system volume is $V_{t}$ and that this approach can be viewed as a simplified transition-state approximation. Nevertheless, the SMM well describes the IMF charge (mass) distributions for thermally driven multifragmentation. Note once again that in the traditional application of the SMM, only one size parameter is used. The shortcoming of such a simplification of the model is obvious now.

The evidence for the existence of two characteristic multifragmentation volumes changes the understanding the time scale of the process (see the bottom of fig.3). One can imagine the following ingredients of the time scale: $t_{1}$ - the mean thermalizataion time of the excited target spectator, $t_{2}-$ the mean time of the expansion to reach the transition state, $t_{3}$ - the mean time up to the multi-scission point. 


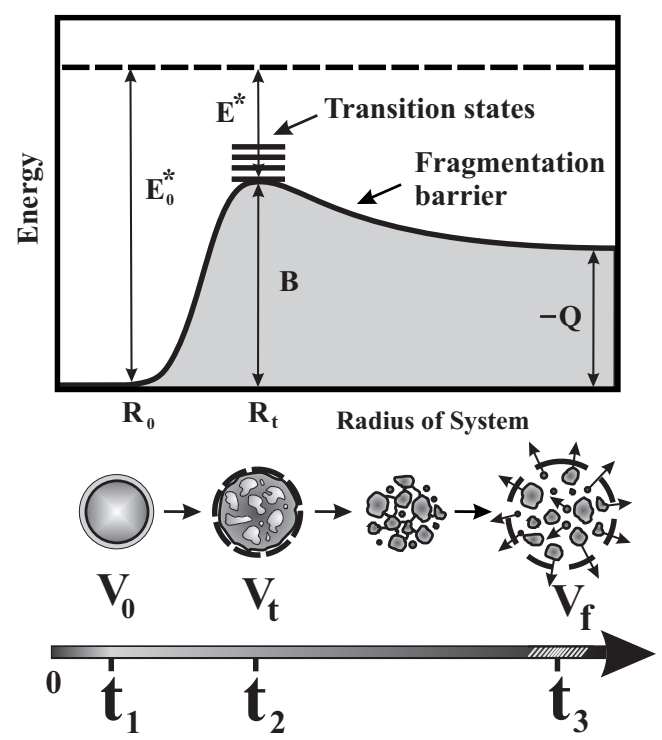

Figure 3: Upper: qualitative presentation of the potential energy of the hot nucleus (with excitation energy $E_{0}^{*}$ ) as a function of the system radius. The ground state energy of the system corresponds to $E=0 . \quad B$ is the fragmentation barrier, $Q$ is the released energy. Bottom: schematic view of the multifragmentation process and its time scale: $t_{1}$ - thermalization time, $t_{2}$ - time of the expansion driven by thermal pressure, $t_{3}$ - the mean time of the multi-scission point (with dispersion, which is measured as fragment emission time, $\left.\tau_{e m}\right)$.

The system configuration on the way to the scission point contains several pre-fragments connected by necks. Their random rupture is characterized by the mean time, $\tau_{n}$, which seems to be a decisive ingredient of the fragment emission time: $\tau_{e m} \approx \tau_{n}$. Formally $\tau_{e m}$ may be understood as the standard deviation of $t_{3}$ :

$$
\tau_{e m}=\left(<t_{3}^{2}>-<t_{3}>^{2}\right)^{1 / 2} .
$$

In the earlier papers, the emission time was related to the mean time of density fluctuations in the system at the stage of fragment formation, at $t \approx t_{2}[19$.

What are the expected values of these characteristic times? Thermalization or energy relaxation time after the intranuclear cascade, $t_{1}$, is model estimated to be (10-20) $\mathrm{fm} / \mathrm{c}$ [20, 21. The Expanding Emitting Source model (EES) predicts $\left\langle t_{2}-t_{1}\right\rangle \approx 70 \mathrm{fm} / \mathrm{c}$ for $p(8.1 \mathrm{GeV})+\mathrm{Au}$ collisions [22]. The model calculation in 23] results in estimation of $t_{3}$ to be (150-200) $\mathrm{fm} / \mathrm{c}$. The only measured temporal characteristic is a fragment emission time, $\tau_{e m}$, which is found in number of papers to be $\approx 50 \mathrm{fm} / \mathrm{c}$ (e.g. see [9]). It would be very important to find a way to measure the value of $t_{3}$.

Note, that in the case of ordinary fission $t_{2}$ is specified by the fission width $\Gamma_{f}$, which corresponds to a mean time of about $10^{-19} \mathrm{~s}$ (or $3.3 \cdot 10^{4} \mathrm{fm} / \mathrm{c}$ ) for an excitation energy of around $100 \mathrm{MeV}$ [24]. The value $\left\langle t_{3}-t_{2}\right\rangle$ is model estimated in a number of papers to be about $1000 \mathrm{fm} / \mathrm{c}$ [25]. A mean neck rupture time, considered as a Rayleigh instability, is estimated in [26] to be: 


$$
\tau_{n}=\left[1.5 \cdot\left(R_{n} / \mathrm{fm}\right)^{3}\right]^{1 / 2} \cdot 10^{-22} s
$$

Generally, the values of $\tau_{n}$ are found to be 200-300 $\mathrm{fm} / \mathrm{c}$ for fission.

Using (1) for the estimation of the mean time for the rupture of the multi-neck configuration in fragmentation, one gets $\tau_{n}$ between 40 and $115 \mathrm{fm} / c$ under the assumption of a neck radius $R_{n}$ between 1 and $2 \mathrm{fm}$. This estimation is in qualitative agreement with the measured values of the fragment emission time $\tau_{e m}$. Evidently, the multifragmentation process is much faster than high energy fission. Table 1 summarizes these estimates.

Table 1 Characteristic times (in $\mathrm{fm} / \mathrm{c}$ ) for fragmentation and fission; the models used are given in brackets; experimental values are marked (Exp).

\begin{tabular}{|c|c|c|c|c|}
\hline & $t_{1}$ & $t_{2}$ & $<t_{3}-t_{2}>$ & $\sigma\left(t_{3}\right)$ \\
\hline Fragmentation & $\begin{array}{c}20 \\
(\mathrm{UU})\end{array}$ & $\begin{array}{c}80 \\
(\mathrm{EES})\end{array}$ & $\begin{array}{c}150 \\
(\mathrm{QMD})\end{array}$ & $\begin{array}{c}50 \pm 10 \\
(\mathrm{Exp})\end{array}$ \\
\hline Fission & & $3 \cdot 10^{4}$ & $\begin{array}{c}2 \cdot 10^{3} \\
(\mathrm{LD})\end{array}$ & $\begin{array}{c}200 \\
(\mathrm{RI})\end{array}$ \\
& & $(\mathrm{Exp})$ & $(\mathrm{T})$ & \\
\hline
\end{tabular}

As for the spatial characteristics, the relative elongation of the very heavy systems $(Z>99)$ at the fission scission point is similar to that for the multi-scission point of medium heavy nuclei. For the fission of the lighter nuclei, (Po-Ac), the scission elongation is larger [26].

A few words about the experimental possibility for finding the total time scale for fragmentation, i.e. the mean value of $t_{3}$. It can be done by the analysis of the fragment-fragment correlation function with respect to relative angle. But, in contrast to the usual IMF-IMF correlation, one of the detected fragments should be the particle ejected during the thermalization time $t_{1}$. For the light relativistic projectiles, it may be the pre-equilibrium IMFs; for heavy-ion induced multifragmentation, a projectile residual (PR) may be used as the trigger related to the initial collision. In the last case the PR-TIMF correlation function should be measured, where TIMF is the intermediate mass fragment from the disintegration of the hot target spectator created via the partial fusion.

\section{Conclusion}

Thermal multifragmentation of hot nuclei is interpreted as the nuclear liquid-fog phase transition inside the spinodal region. Experimental evidence is presented for the existence of two characteristic volumes for the process: transition state and kinetic freeze-out volumes. This is similar to that for ordinary fission. The dynamics is similar also for the two processes, but multifragmentation is much 
faster than high energy fission. The IMF emission time is related to the mean rupture time at the multi-scission point, which corresponds to the freeze-out configuration.

The authors are grateful to A. Hrynkiewicz, A.I. Malakhov, A.G. Olchevsky for support and to I.N. Mishustin and W. Trautmann for illuminating discussions. The research was supported in part by the Russian Foundation for Basic Research, Grant 06-02-16068, the Grant of the Polish Plenipotentiary to JINR, Bundesministerium für Forschung und Technologie, Contract No 06DA453.

\section{References}

[1] BOTVINA A.S. ET AL., Yad. Fyz. 42 (1985) 1127;

BONDORF J.P. ET AL., Phys. Rep. 257 (1995) 133.

[2] GROSS D.H.E., Rep. Progr. Phys. 53 (1990) 605.

[3] SAUER G., Chandra H. AND MOSEl U., Nucl. Phys. A264 (1976) 221.

[4] JAQAman H., MEKJiAn A.Z. AND ZAMICK L., Phys. Rev., C 27 (1983) 2782.

[5] SIEMENS P.J., Nature, 305 (1983) 410; Nucl. Phys. A428 (1984) 189c.

[6] KARNAUKHOV V.A., Phys. of At. Nuclei 62 (1997) 237.

[7] KARNAUKHOV V.A. ET AL., Nucl. Phys, A734 (2004) 520.

[8] KARNAUKHOV V.A. ET AL., Nucl. Phys. A749 (2005) 65c.

[9] RODIONOV V.K. ET AL., Nucl. Phys. A700 (2002) 457.

[10] LOPEZ J.A. AND RANDRUP J., Nucl. Phys. A503 (1989) 183.

[11] LOPEZ J.A. AND RANDRUP J., Nucl. Phys. A512 (1990) 345.

[12] BAO-AN Li ET AL., Phys. Lett. B 335 (1994)1.

[13] NATOWITZ J.B., ET AL., Phys. Rev. C 66 (2002) 031601(R).

[14] D'AGostino M., ET AL., Nucl. Phys. A699 (2002) 795.

[15] VIOLA V.E., Nucl. Phys. A734 (2004) 487.

[16] RADUTA AD.R., BORDERIE B. ET AL., Phys. Lett. B 623 (2005)43.

[17] ELliOTt J.B. ET AL., Phys. Rev. Lett. 88 (2002) 042701.

[18] ElliotT J.B. ET AL., Phys. Rev. C 67 (2003) 024609.

[19] WANG G. ET AL., Phys. Rev. C 48 (1998) R2786.

[20] CASSING W., Z. Phys. A 327 (1987) 447.

[21] BORDERIE B., Preprint Orsay/IPNO-DRE-92-03, Preprint, 1992.

[22] AVDEYEV S.P. ET AL., Eur. J. Phys. A 3 (1998) 75.

[23] BARAN V. ET AL., Nucl. Phys. A703 (2002)603.

[24] GOldenBaum F. ET AL., Phys. Rev. C 82 (1999) 5012.

[25] HILSCHER D., H. ROSSNER H., Annales de Phys. 17 (1992) 471.

[26] BROSA U. ET AL., Phys. Rep. 197 (1990) 162. 